\title{
PERK-elF2a-ATF4 signaling contributes to osteogenic differentiation of periodontal ligament stem cells
}

\author{
Shuangyan Yang ${ }^{1} \cdot$ Lihua $^{\mathrm{Hu}^{2}} \cdot$ Chunling Wang $^{1} \cdot$ Fulan Wei $^{1}$
}

Received: 30 December 2019 / Accepted: 25 February 2020 / Published online: 2 March 2020

(c) The Author(s) 2020

\begin{abstract}
Protein kinase-like endoplasmic reticulum kinase (PERK) is a type I transmembrane protein located in the endoplasmic reticulum (ER). The PERK-eukaryotic initiation factor $2 \alpha$ (eIF2 $\alpha$ )-activating transcription factor 4 (ATF4) pathway has been proved to be involved in osteoblast differentiation, but the involvement of the PERK-eIF2 $\alpha$-ATF4 signaling pathway in osteogenic differentiation of human periodontal ligament stem cells (hPDLSCs) has remained unclear. Therefore, the aim of this study was to explore the role of PERK in osteogenic differentiation of hPDLSCs and to assess whether PERK-eIF2 $\alpha$-ATF4 contributes to the process of osteogenic differentiation in hPDLSCs. In our study, we constructed PERK-overexpressed and PERK-silenced hPDLSCs by lentiviral transduction. Furthermore, lentivirus-transfected cells were induced to differentiate into osteoblast cells for different days. Alkaline phosphatase (ALP) activity and Alizarin Red staining were used to evaluate the mineralization capacity, and the expression levels of related genes-ATF4, ALP, bone sialoprotein, runt-related transcription factor 2 (Runx2), and osteocalcin were measured to evaluate the osteogenic differentiation of hPDLSCs. The results showed that over-expression of PERK greatly increased ALP activity and the expression levels of related osteogenic genes, which displayed the strongest osteogenesis capacity. However, suppression of PERK caused decreased ALP activity and the weakest osteogenesis capacity, and the levels of ATF4 and p-eIF2 $\alpha$ in PERK-silenced hPDLSCs were also decreased. Our results indicated that the PERK gene plays an important role in the differentiation of hPDLSCs to osteoblast-like cells. The PERK-eIF2 $\alpha$-ATF4 signaling pathway contributes to osteoblast differentiation of hPDLSCs.
\end{abstract}

Keywords Protein kinase-like endoplasmic reticulum kinase $\cdot$ Activating transcription factor-4 $\cdot$ Osteogenic differentiation · Human periodontal ligament stem cells

\section{Introduction}

The periodontal ligament (PDL) is a dense connective tissue around the root and the alveolar bone. PDL plays an important role not only in maintaining the homeostasis of the periodontal tissue but also in bone tissue remodeling, healing, and regeneration. Periodontal ligament stem cells

Fulan Wei

weifl@sdu.edu.cn

1 Department of Orthodontics, School and Hospital of Stomatology, Shandong University \& Shandong Key Laboratory of Oral Tissue Regeneration \& Shandong Engineering Laboratory for Dental Materials and Oral Tissue Regeneration, No. 44-1 Wenhua Road West, Jinan 250012, Shandong, China

2 Department of Implantology, Shandong Provincial Hospital, Shandong University, Jinan, People's Republic of China
(PDLSCs) have been successfully isolated from the PDL (Seo et al. 2004). They have been proved to have multiple differentiation potential; thus, they can differentiate into osteoblasts, cementoblasts, adipose tissue, cartilage, and provide new prospects for tissue regeneration application (Gay et al. 2007; Kim et al. 2012; Nagatomo et al. 2006; Silvério et al. 2010). Despite the interest in the clinical application of PDLSCs, the molecular mechanism controlling differentiation remains unclear, which constrains their clinical application.

Activating transcription factor 4 (ATF4) is a known transcription factor involved in osteoblast differentiation and preservation of mature osteoblast function. From the composition, ATF4 belongs to the family of cAMP-responsive element binding proteins (CREB) (Hai and Hartman 2001; Xiao et al. 2005; Yang and Karsenty 2004). Knockout of ATF4 in mice leads to severe osteoporosis, osteodysplasty, and disorders of bone mineralization, which are caused by 
the inability of osteoblasts to completely differentiate and to accurately function. Moreover, ATF4 prevents the synthesis of type I collagen, the most abundant extracellular protein of osteoblasts (Komori 2006). Furthermore, it has been shown that ATF4 is involved in the differentiation of PDLSCs to osteoblast-like cells (Wei et al. 2008a). These studies fully indicated that ATF4 is a critical transcription factor in osteoblast differentiation and bone development. However, ATF4 is a downstream factor and a translational target of protein kinase-like endoplasmic reticulum kinase (PERK). PERK is a major transducer of the unfolded protein response in the endoplasmic reticulum (ER), and it can transduce the signals from the ER to the cytosol or nucleus to avoid accumulation of excessive unfolded proteins in the ER. Activation of PERK causes phosphorylation of the $\alpha$-subunit of the eukaryotic initiation factor 2 (eIF $2 \alpha$ ), which promotes ATF4 translation. PERK-deficient mice exhibited severe osteoporosis, and some mice showed neonatal developmental deficiency, including skeletal hypo-development and growth stunting (Harding et al. 2000; Huang et al. 2006; Iyer et al. 2004; Wei et al. 2008b). These observations occur due to reduction of the number of osteoblasts and impaired function of osteoblast differentiation. The changes in bone tissue noted in PERK-deficient mice are similar to those in ATF4-deficient mice. Recent studies found that the level of ATF4 was sharply declined in PERK-knockout osteoblasts. Moreover, alkaline phosphatase activity was decreased and mineralized nodule formation was delayed in $\mathrm{PERK}^{-/-}$osteoblasts. These results indicated that activation of the PERKeIF2 $\alpha$-ATF4 signaling pathway promoted the expression of some genes that are essential for osteogenesis. However, the involvement of the PERK-eIF2 $\alpha$-ATF4 pathway in osteogenic differentiation of PDLSCs has remained poorly understood.

To investigate this point, we first constructed PERKsilenced and PERK-overexpressed hPDLSCs by lentiviral transduction. Then, we explored whether and how the expression of ATF4 is affected after PERK transfection of hPDLSCs, and whether PERK-eIF2 $\alpha$-ATF4 contributes to the process of differentiation of hPDLSCs into osteoblastlike cells. Our results provide novel insights into the mechanism that underlies the osteogenic differentiation of PDLSCs and could serve as a stepping stone for the development of novel therapeutic strategies that can be used to regenerate dental tissues.

\section{Materials and methods}

\section{Cell culture and isolation of hPDLSCs}

Human PDLSCs were obtained from healthy PDL tissue of premolar teeth, extracted from young men (11-16 years old) for orthodontic reasons. Informed consent was obtained from the donors and their parents. The PDL tissues were scraped from the middle third of the roots and transferred to plastic culture dishes. Then the explants were cultured in alpha minimum essential medium ( $\alpha$-MEM; Gibco, USA) supplemented with $100 \mathrm{U} / \mathrm{ml}$ penicillin, $100 \mathrm{mg} / \mathrm{ml}$ streptomycin, and $15 \%(\mathrm{v} / \mathrm{v})$ heat-inactivated fetal bovine serum (FBS; Hyclone, USA), at $37^{\circ} \mathrm{C}$ in a humidified atmosphere of $95 \%$ air and $5 \% \mathrm{CO}_{2}$. When the cells started to grow out from the explants, the medium was replaced every 2-3 days. Upon reaching confluence, the cells were separated and subcultured with $0.25 \%$ trypsin. For the experiments, the third or fourth passage hPDLSCs were used.

\section{Cell transduction mediated by lentiviral vector}

The lentivirus vector for PERK overexpression (LentiPERK), PERK inhibition (anti-PERK), and empty lentivirus vector LV-GFP (PERK control or anti-PERK inhibitor control) for negative control was constructed by Genechem Biological Company. We conducted a preliminary experiment to determine the optimal value of multiplicity of infection (MOI). After the cultures reached $40-60 \%$ confluence, hPDLSCs were infected with lentivirus at MOI of 30, 50, 60,80 , and 100 . Then the transfection efficiency was evaluated using a fluorescence microscope (Nikon, Japan). In the formal experiment, we added the appropriate amounts of lentivirus according to the appropriate MOI values (LentiPERK: $\mathrm{MOI}=100$, anti-PERK: $\mathrm{MOI}=100$, Empty vector: $\mathrm{MOI}=60$ ). To promote the transfection efficiency, $5 \mu \mathrm{g} /$ $\mathrm{ml}$ polybrene was added to the positively transfected cells according to the manufacturer's instructions.

\section{Osteogenic induction}

hPDLSCs were plated at $3 \times 10^{4} \mathrm{~cm}^{2}$ in 6 -well plates and cultured for 2-3 days. Then the medium was changed to an osteogenic inducing medium $(\alpha$-MEM containing $10 \%$ FBS, $100 \mu \mathrm{M}$ L-ascorbate-2-phosphate, $10 \mathrm{mM}$ $\beta$-glycerophosphate, and $10 \mathrm{nM}$ dexamethasone) (SigmaAldrich Corp., St. Louis, MO, USA) to induce osteogenesis. Control groups were treated with $\alpha$-MEM containing $10 \%$ FBS and $1 \%$ antibiotics only. Cells were cultured up to the indicated days in the presence of osteogenic differentiation medium.

\section{Alkaline phosphatase and alizarin red detection}

After osteogenic induction for 7 days, hPDLSCs were studied to determine their alkaline phosphatase (ALP) activity. According to the manufacturer's protocol (Sigma-Aldrich Corp., St. Louis, MO, USA), the cells were washed with PBS and fixed with $4 \%$ paraformaldehyde for $15 \mathrm{~min}$. After 
that, cells were stained with a mixed solution of $0.25 \%$ naphthol AS-BI phosphate and $0.75 \%$ fast red violet (from the ALP activity kit). The ALP activity was normalized to the cellular protein concentration in the sample using the ALP activity kit.

To detect mineralization, cells were induced to osteodifferentiate for 3 weeks. The cells were washed with PBS and fixed for 15 min with $70 \%$ methanol. Then the cells were stained with 2\% Alizarin Red solution (pH 4.2) (SigmaAldrich Corp., St. Louis, MO, USA) for $10 \mathrm{~min}$ at room temperature. After that, cells were washed with tap water several times. Images of the stained cells were captured using a digital camera (Leica, Germany) and microscope (Nikon). To quantitatively determine calcium, Alizarin Red was destained with $10 \%$ cetylpyridinium chloride in $10 \mathrm{mM}$ sodium phosphate for $30 \mathrm{~min}$ at room temperature. Aliquots of each sample were measured at $562 \mathrm{~nm}$ and compared to a standard calcium curve with calcium dilutions in the same solution. The final calcium level in each group was normalized to the total protein concentration detected in duplicate plates.

\section{Quantitative real-time RT-PCR}

To measure the mRNA levels of osteogenic transcriptional factors, real-time RT-PCR was performed. The total RNA was isolated from cultured cells under different groups using Trizol reagent (TAKARA Bio Inc., Otsu, Shiga, Japan). After RNA extraction, $1 \mu \mathrm{g}$ of total RNA was used for cDNA synthesis using the PrimeScript ${ }^{\circledR}$ RT reagent Kit with gDNA Eraser (TAKARA Bio Inc., Otsu, Shiga, Japan). Real-time PCR was performed using Roche Light Cycler 480 detection system in a $20 \mu \mathrm{l}$ reaction volume per tube containing $2.0 \mu \mathrm{l} \mathrm{cDNA}$ and $10 \mu \mathrm{l}$ SYBR Premix Ex Taq (TAKARA Bio Inc., Otsu, Shiga, Japan). The standard PCR conditions were as follows: $95^{\circ} \mathrm{C}$ for $30 \mathrm{~s}$, then 40 cycles of $95^{\circ} \mathrm{C}$ for $5 \mathrm{~s}, 60^{\circ} \mathrm{C}$ for $20 \mathrm{~s}$ after a hot start, and $30 \mathrm{~s}$ at $60^{\circ} \mathrm{C}$. The specificity of the reaction is given by the detection of the melting temperatures (Tms) of the amplification products immediately after the last reaction cycle. The value obtained from $2^{-\Delta \Delta \mathrm{CT}}$ was used for comparative quantization. All of the PCRs were performed in quintuplicate. A housekeeping gene GAPDH was used as an internal control to quantify and normalize the results. Gene-specific primers used are shown in Table 1.

\section{Western blotting}

To investigate the protein levels of the osteogenic markers, western blot analysis was used. Cells were collected and washed once with ice-cold phosphate-buffered saline (PBS), and then cells were placed in protein lysates for $30 \mathrm{~min}$ on ice. The supernatant was obtained by centrifugation at
Table 1 The primers used for quantitative RT-PCR analysis

\begin{tabular}{lll}
\hline Gene name & Primer $\left(5^{\prime}\right.$ to $\left.3^{\prime}\right)$ & Primer sequence \\
\hline PERK & Forward & TCTTGGTTGGGTCTGATGAAT \\
& Reverse & GATGTTCTTGCTGTAGTGGGGG \\
ATF4 & Forward & GTCTCCGTGAGCGTCCAT \\
& Reverse & CAGAAGCCAACTCCCATTAG \\
OCN & Forward & TAGTGAAGAGACCCAGGCGCT \\
& Reverse & ATAGGCCTCCTGAAAGCCGA \\
ALP & Forward & GGACCATTCCCACGTCTTCAC \\
& Reverse & CCTTGTAGCCAGGCCCATTG \\
BSP & Forward & GTCACTGGAGCCAATGCAGAA \\
& Reverse & CCCACCATTTGGAGAGGTTGT \\
Runx2 & Forward & CGAATAACAGCACGCTATTAA \\
& Reverse & GTCGCCAAACAGATTCATCCA \\
GAPDH & Forward & TCATGGGTGTGAACCATGAGAA \\
& Reverse & GGCATGGACTGTGGTCATGAG \\
\hline
\end{tabular}

$12,000 \mathrm{rpm}$ for $15 \mathrm{~min}$ at $4{ }^{\circ} \mathrm{C}$. The protein was quantified using the BCA Protein Assay kit (Sunbio Biological Technology CO Ltd, Beijing, China) according to the manufacturer's protocol. Proteins were separated by electrophoresis on $10 \%$ SDS -PAGE gels with a constant voltage of $80 \mathrm{~V}$ for $2 \mathrm{~h}$. The separated proteins were transferred electrophoretically onto polyvinyldifluoride (PVDF) membranes (Amersham Biosciences, Uppsala, Sweden). The membranes were blocked for 45-90 min at room temperature with 5\% (v/v) skimmed milk in Tris-buffered saline Tween-20 (TBS-T), and then incubated at $4{ }^{\circ} \mathrm{C}$ overnight with primary antibodies. After three 5-min washes in TBS-T, the membranes were incubated at room temperature for $2 \mathrm{~h}$ with biotinylated goat anti-mouse IgG (1:3000, Zhong Shan-Golden Bridge Biological Technology Co. Ltd., Beijing, China). Then the membranes received three 5-min washes in TBS-T. The immune reactive bands were detected using the ECL chemiluminescence reaction (Amersham Pharmacia Biotech, Inc., USA) according to the manufacturer's instructions. Images were scanned, and the densitometric value of each band was determined by LabWorks Image (Scion Corporation, Frederick, MD, USA). Loading differences were normalized by assessing the housekeeping protein in each sample. The primary antibodies used were as follows: anti-eIF2 $\alpha$ (Cell Signaling; 1:1000), anti-phospho eIF2 $\alpha$ (Stress Gen; 1:1000), anti-ATF4 (Abcam; 1:500), and anti-GAPDH (Santa Cruz Biotechnology; 1:3000).

\section{Statistical analysis}

For statistical analysis, Student's $t$ test and one-way ANOVA were used. Data are expressed as the mean $\pm \mathrm{SD}$. Differences at $p<0.05$ were considered to be significant. 


\section{Results}

\section{PERK expression increased during osteogenic differentiation of hPDLSCs}

After about 1-2 weeks, primary hPDLSCs gradually grew out from the scraped PDL tissue block (Fig. 1a). After passage, the cells were evenly distributed, and they grew in a spiral or radial arrangement. Figure $1 \mathrm{~b}$ shows the third passage of hPDLSCs.

Osteoblast differentiation of hPDLSCs was induced by standard osteoblast-induction medium, and osteogenic differentiation of hPDLSCs was evidenced by increased expression of genes associated with osteogenic differentiation, alkaline phosphatase (ALP), bone sialoprotein (BSP), runt-related transcription factor 2 (Runx2), and osteocalcin (OCN) at days 1, 3, 7, 10, and 14 after induction (Fig. 1c-f). Increased expression of osteoblast-associated genes was consistent with previous research findings describing hPDLSC differentiation into osteoblasts (Seo et al. 2004; Silvério et al. 2010).

Next, we determined the changes in PERK expression during osteogenic differentiation using RT-PCR. The result showed that PERK expression was up-regulated in a time-dependent manner after the induction of osteogenic differentiation of hPDLSCs, which was remarkably increased compared to the undifferentiated hPDLSCs (0 day) (Fig. 1g). The results indicated that PERK might play a role during osteogenic differentiation.

\section{Overexpression of PERK increased the osteogenic differentiation of hPDLSCs}

To assess the effect of PERK on osteogenic differentiation of hPDLSCs, we asked Genechem Company to prepare a lentiviral construct for overexpression of PERK and its control. After transfection, most hPDLSCs (>90\%) expressed the green fluorescent protein (GFP) and exhibited a morphology similar to that of PERK control-infected hPDLSCs (Fig. 2a).

After infection, hPDLSCs were induced to differentiate into osteoblasts for different days, and then ALP and Alizarin Red, and expression of osteogenic differentiation markers were detected. One week after induction, ALP activity in PERK-transfected cells showed a significant increase when compared to that in untransfected or PERK control-transfected cells (Fig. 2b, c). After culturing hPDLSCs in osteogenesis-inducing medium for 21 days, Alizarin Red staining and quantitative calcium measurements revealed that matrix mineralization was increased in PERK cells than in PERK control-infected hPDLSCs and uninfected hPDLSCs (Fig. 2b-d), which indicated that PERK overexpression significantly promoted matrix mineralization in hPDLSCs.

Consistent with this finding, real-time RT-PCR results showed that the expression levels of all of the osteogenic marker genes measured (ALP, BSP, OCN, and Runx2) were remarkably increased following infection by PERK. There was no significant difference in the mRNA expression levels of all of the osteogenic related genes between PERK controltransfected cells and non-transfected cells (Fig. 2e-h).

\section{Inhibition of PERK decreased the osteogenic differentiation of hPDLSCs}

To further assess the effect of PERK on osteogenic differentiation of hPDLSCs, anti-PERK-transfected hPDLSCs and anti-PERK control-transfected cells were induced to differentiate along osteogenic lineages. After transfection, most hPDLSCs (>90\%) expressed the green fluorescent protein (GFP) and exhibited a morphology similar to that of antiPERK control-infected hPDLSCs (Fig. 3a).

Conversely, after one week of induction, the ALP activity in anti-PERK transfected cells was markedly decreased compared to that in the control group and the anti-PERKtransfected group (Fig. 3b, c). Moreover, Alizarin Red staining and quantitative calcium measurements indicated that mineralization was also significantly lower in anti-PERK cells than in anti-PERK control-infected hPDLSCs and uninfected hPDLSCs after three weeks of induction (Fig. 3b-d).

Real-time RT-PCR results showed that the mRNA levels of osteogenesis-related genes were lower in anti-PERKtransfected cells (Fig. 3e-h). There were significant differences when compared to the other two groups.

\section{PERK-elF2 $a-A T F 4$ signaling pathway played a role during osteogenic differentiation of hPDLSCs}

To determine whether the PERK-eIF2 $\alpha$-ATF4 pathway was involved in osteogenic differentiation of hPDLSCs, PCR and western blot were performed to examine the related genes.

The expression of ATF4 mRNA in lentivirus-transfected cells at 7 days after induction was assessed by real-time RT-PCR. ATF4 mRNA expression levels were up-regulated after transfection with PERK overexpression. However, the expression levels of ATF4 were markedly decreased in antiPERK transfected hPDLSCs ( $<<0.01$; Fig. 4a).

Western blotting analysis revealed the protein levels of total-eIF2 $\alpha$ (t-eIF2 $\alpha$ ), phospho-eIF2 $\alpha$ (p-eIF $2 \alpha$ ), and ATF4 in lentivirus-transfected cells at 7 days after induction. The protein levels of ATF4 and p-eIF2 $\alpha$ were up-regulated after transfection with PERK overexpression, while inhibition of PERK decreased the protein levels of p-eIF2 $\alpha$ and ATF4 $(\mathrm{p}<0.01$; Fig. $4 \mathrm{~b}-\mathrm{e})$. We also assessed total eIF $2 \alpha$ (t-eIF $2 \alpha)$, 
A

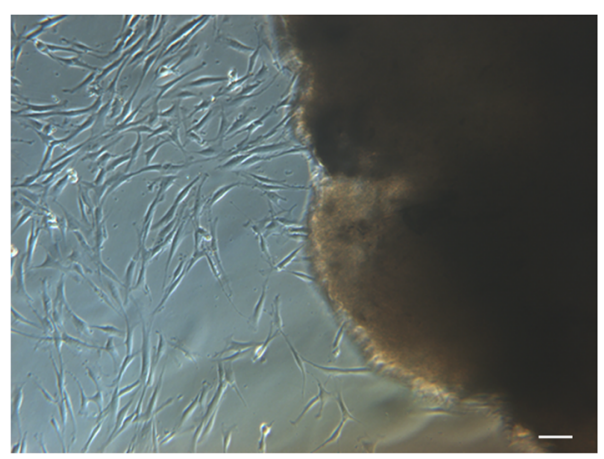

C

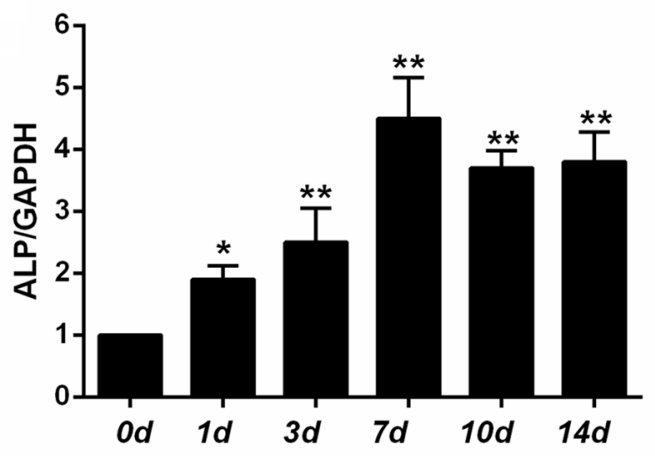

$\mathbf{E}$

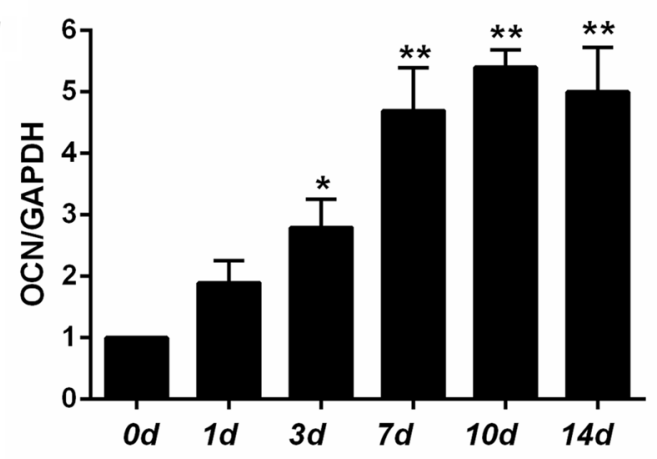

B

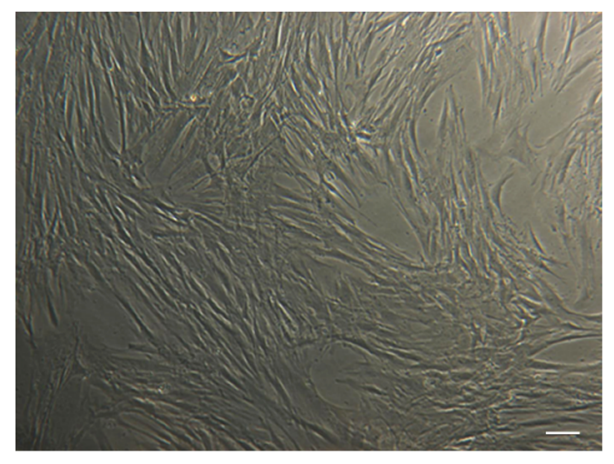

D

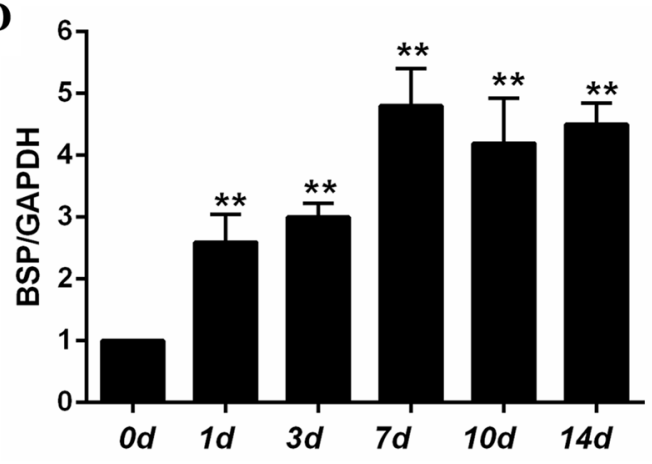

F

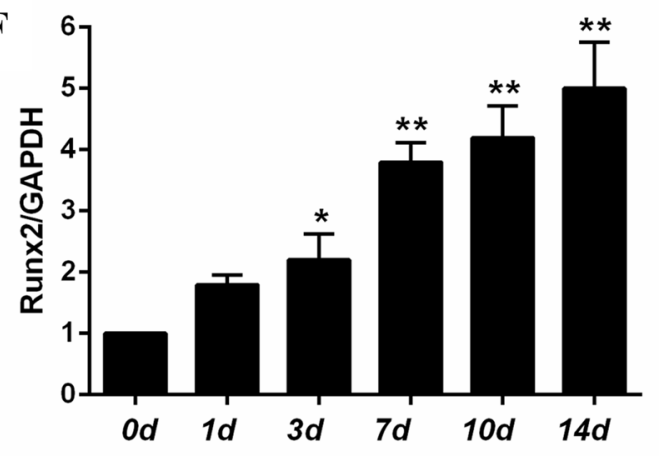

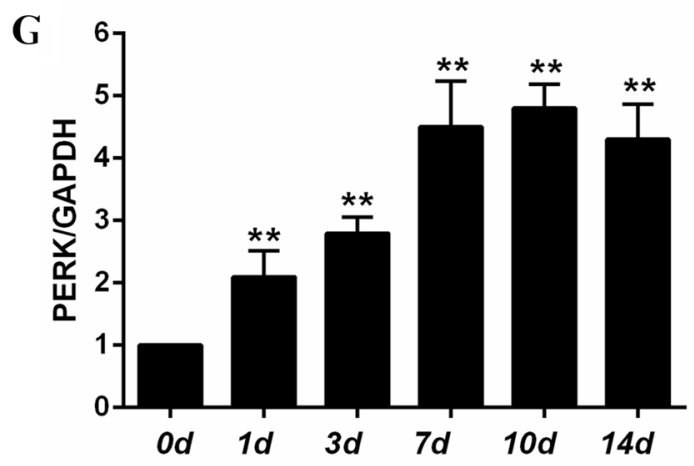

Fig. 1 PERK was up-regulated during osteogenic differentiation of hPDLSCs. Osteogenic differentiation of hPDLSCs was induced for 14 days. Samples were prepared at days $0,1,3,7,10$, and 14 after the induction and used immediately for real-time PCR analysis. a Primary hPDLSCs were cultured successfully by tissue explant technique, and they grew out from the scraped PDL tissue. b The third passage of hPDLSCs (magnification, $\times 100$ ). $\mathbf{c}-\mathbf{f}$ The expression lev- els of osteoblast marker genes, including ALP (c), BSP (d), Runx2 (e), and OCN (f), were analyzed by qRT-PCR during osteoblast differentiation. GAPDH was used as an internal standard. $\mathbf{g}$ The PERK level was increased in hPDLSCs after osteogenic induction. All of the experiments were performed in triplicate, and the results are expressed as mean $\pm \mathrm{SD}$. $* \mathrm{p}<0.05, * * \mathrm{p}<0.01$ compared with the corresponding control sample prepared at the same time point 
A

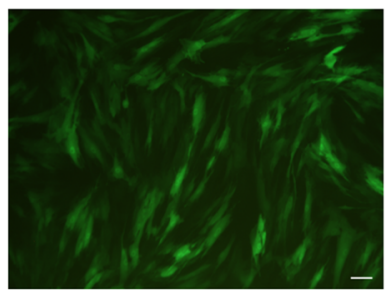

Lenti-Cont

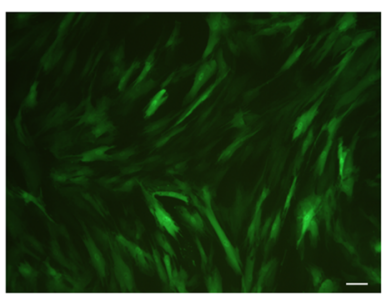

Lenti-PERK

C

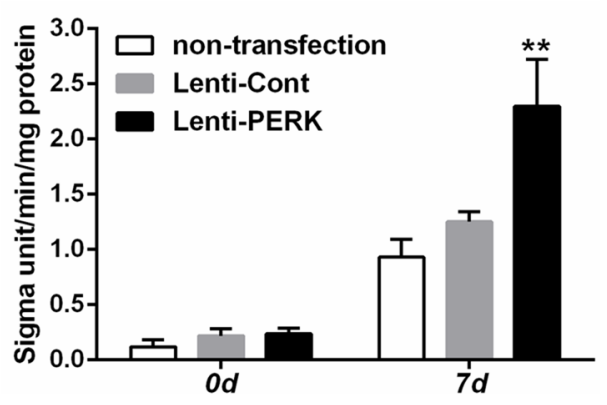

$\mathbf{E}$

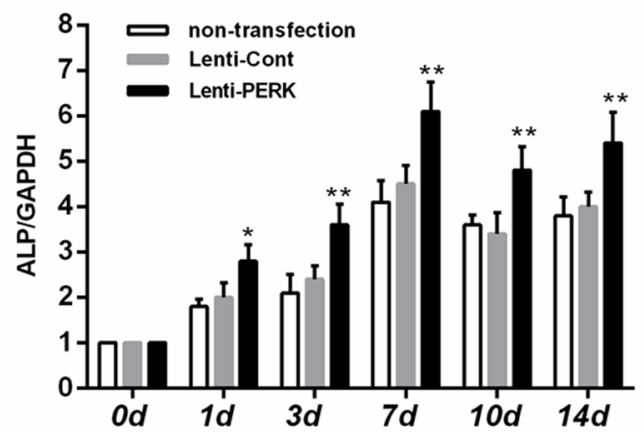

G

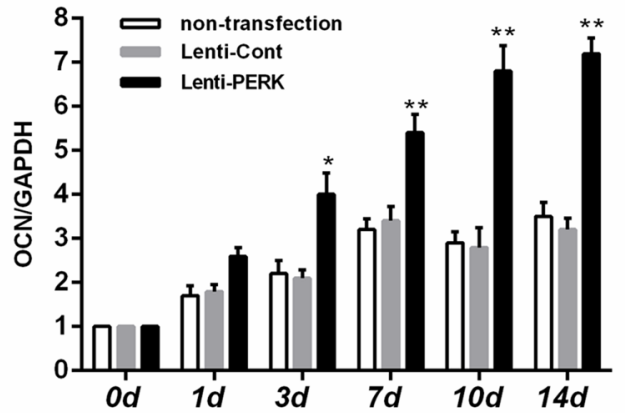

Fig. 2 Overexpression of PERK promoted osteogenic differentiation of hPDLSCs. a Fluorescence microscopic analysis of hPDLSCs transfected with the empty vector (left, the Lenti-Cont group) or PERK overexpression (right, Lenti-PERK group), showing no obvious difference in their morphology (magnification, $\times 200$ ). b ALP staining and Alizarin Red S staining in the three experimental groups.

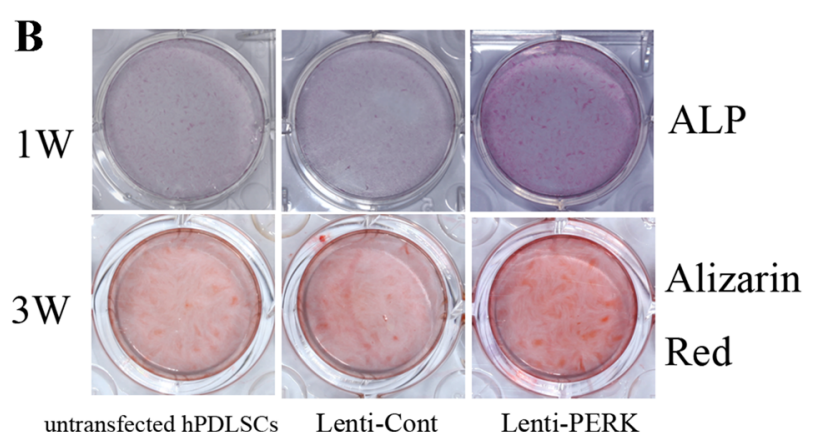

D

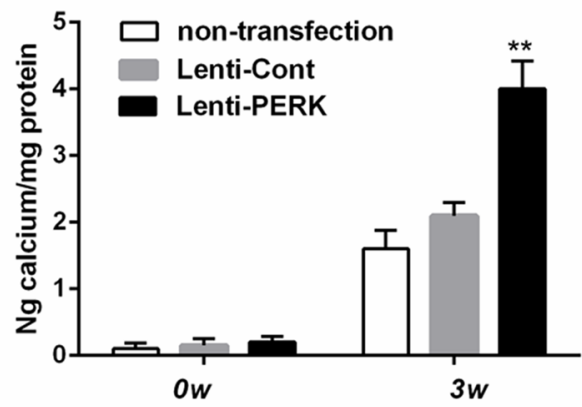

$\mathbf{F}$

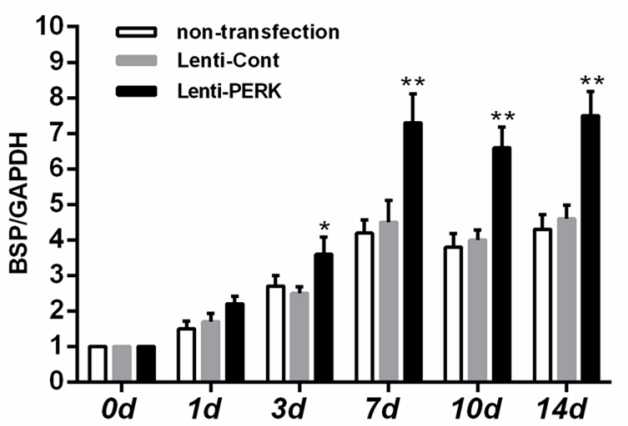

$\mathbf{H}$

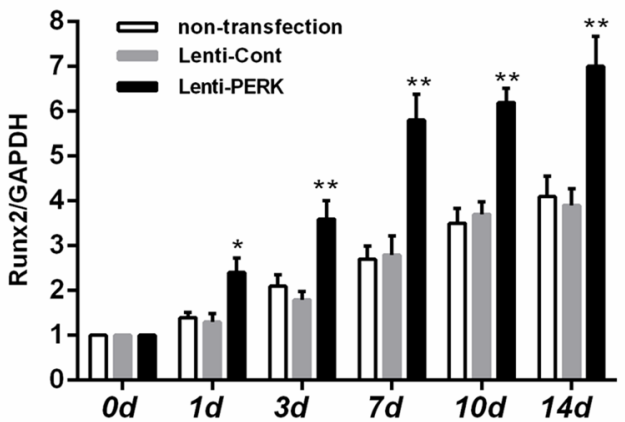

c and d Quantitation of ALP (c) and calcium (d) levels. e-h Realtime RT-PCR analysis of the expression levels of ALP (e), BSP (f), Runx2 (g), and OCN (h) in the three experimental groups. ${ }^{*} \mathrm{p}<0.05$, $* * \mathrm{p}<0.01$ compared with non-transfected hPDLSCs. Scale bar indicates a distance of $100 \mu \mathrm{m}$ 


\section{A}

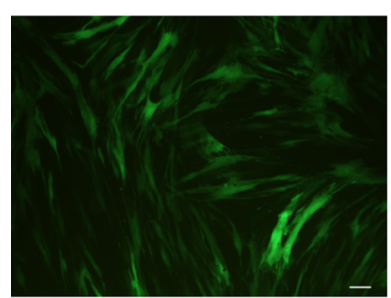

anti-Cont

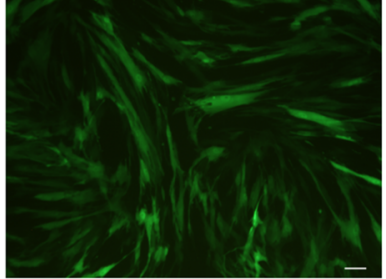

anti-PERK
C

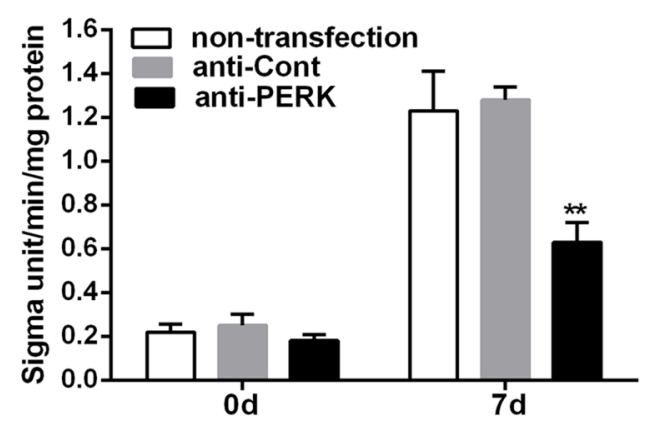

E

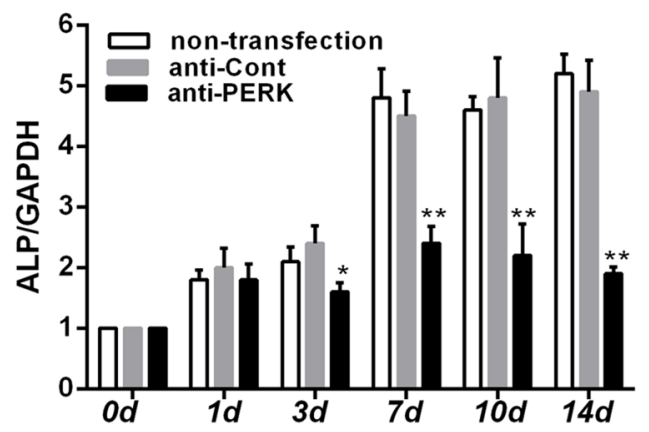

G

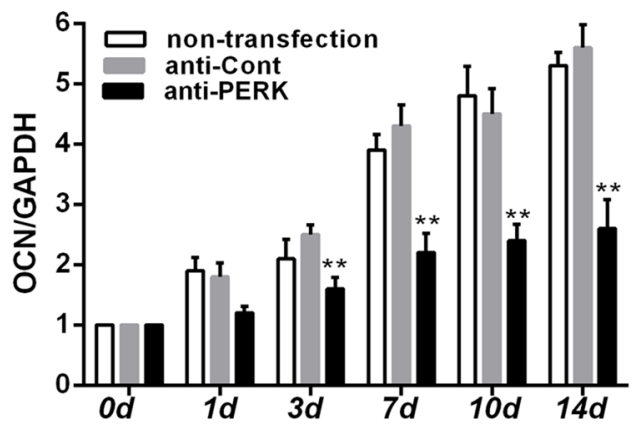

Fig. 3 Down-regulation of PERK inhibited osteogenic differentiation of hPDLSCs. a Fluorescence microscopic analysis of hPDLSCs transfected with the empty vector (left, the anti-Cont group) or PERK inhibition (right, the anti-PERK group), showing no obvious difference in their morphology (magnification, $\times 200$ ). b ALP staining and Alizarin Red S staining in the three experimental groups. $\mathbf{c}$ and

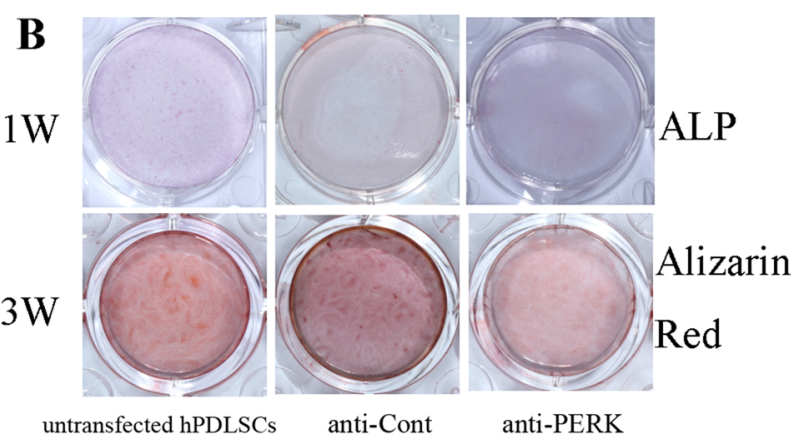

D

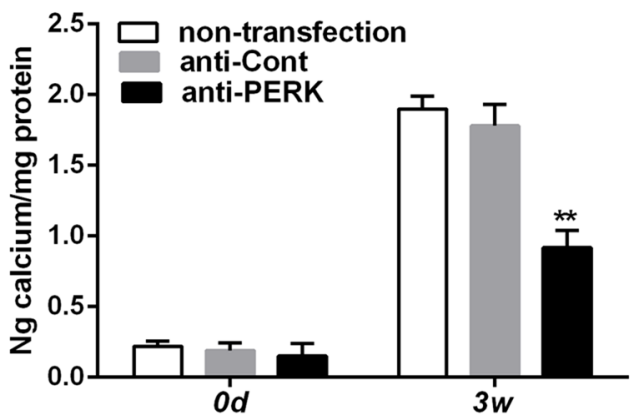

F

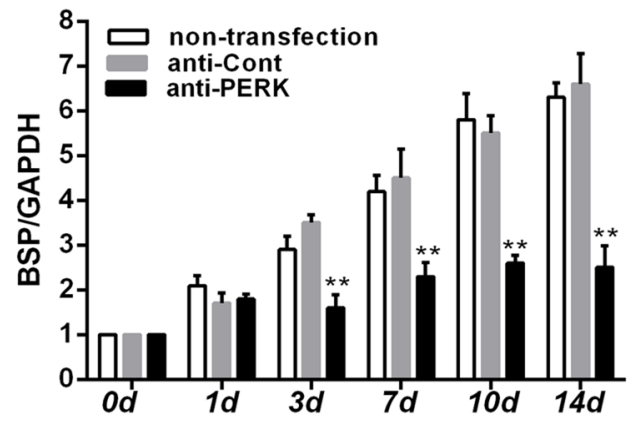

H

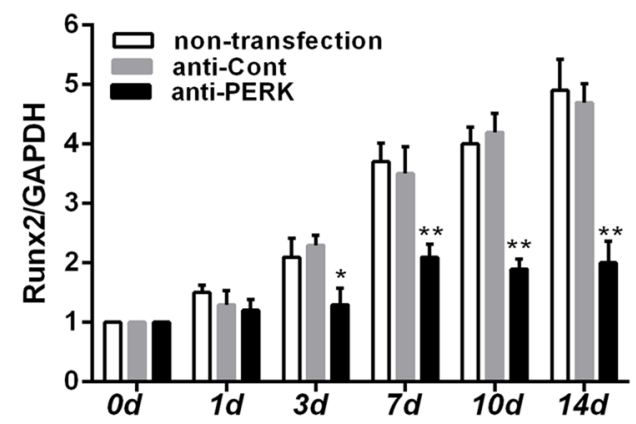

d Quantitation of ALP (C) and calcium d levels. e-h Real-time RTPCR analysis of the expression levels of ALP (e), BSP (f), Runx2 (g), and $\mathrm{OCN}(\mathbf{h})$ in the three experimental groups. $* \mathrm{p}<0.05, * * \mathrm{p}<0.01$ compared with non-transfected hPDLSCs. Scale bar indicates a distance of $100 \mu \mathrm{m}$ 
A

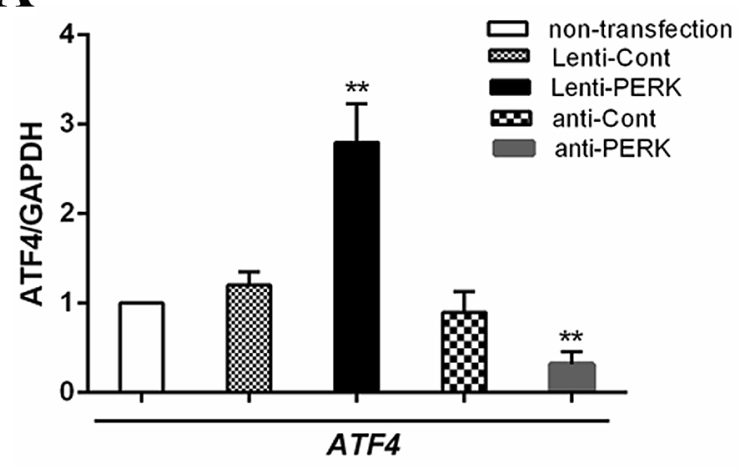

C

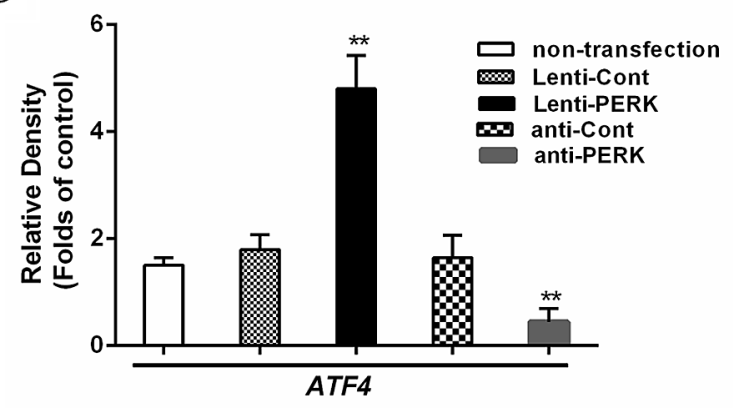

B<smiles>[TeH2]=[Pt+2]</smiles>

ATF4

p-eIF $2 \alpha$

GAPDH

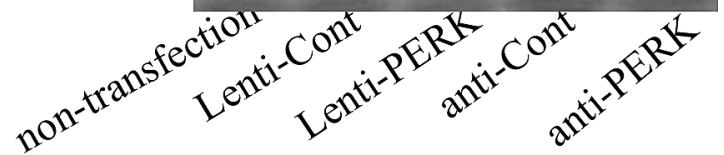

D

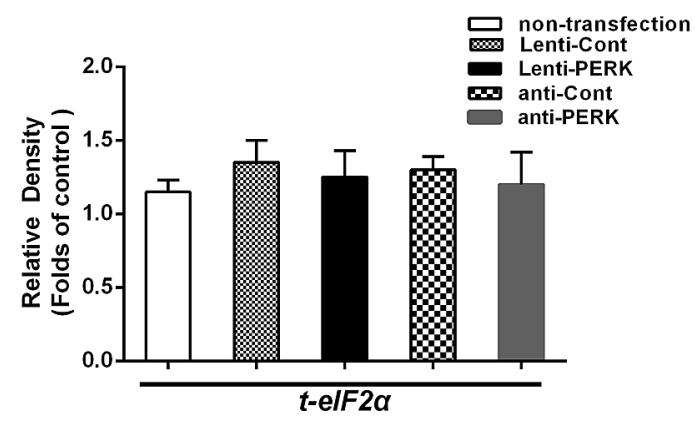

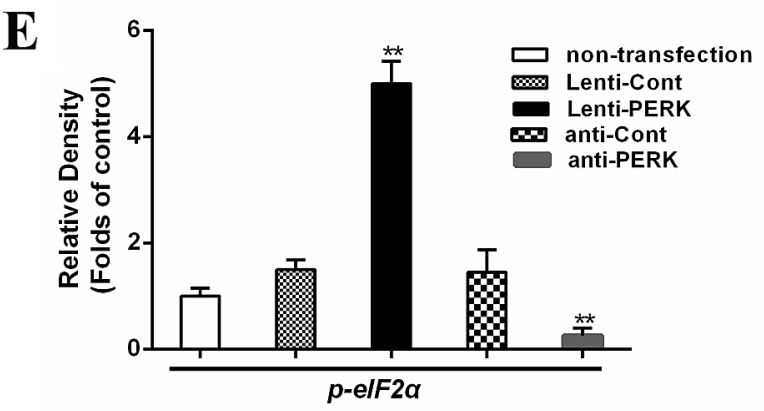

Fig. 4 PERK-eIF2 $\alpha$-ATF4 signaling pathway played a role during osteogenic differentiation of hPDLSCs. a The expression of ATF4 mRNA in lentivirus-transfected cells at 7 days after induction was assessed by qRT-PCR. ATF4 mRNA expression levels were up-regulated after transfection with PERK overexpression. However, the expression of ATF4 was markedly decreased in anti-PERK transfected hPDLSCs. b Western blotting analysis of untransfected cells and lentivirus-transfected cells after 7 days of osteogenic induction.
The expression levels of ATF4 and phospho-eIF $2 \alpha$ (p-eIF2 $\alpha$ ) proteins were up-regulated after transfection with PERK overexpression. In contrast, these proteins were severely inhibited in the anti-PERK group. The expression of total-eIF $2 \alpha$ (t-eIF $2 \alpha$ ) showed no obvious changes. $\mathbf{c}-\mathbf{e}$ The histogram shows the quantitative analysis of the expression levels of ATF4, p-eIF2 $\alpha$, and t-eIF $2 \alpha$ proteins. ${ }^{*} \mathrm{p}<0.05$, $* * \mathrm{p}<0.01$ compared with untransfected hPDLSCs

the PERK-eIF2 $\alpha$-ATF4 signaling pathway plays a role during osteogenic differentiation of hPDLSCs. 


\section{Discussion}

As already known, PDLSCs have multipotential capability to differentiate into osteogenic, adipogenic, and chondrogenic tissues (Kim et al. 2012). They have been shown to exhibit biological properties similar to undifferentiated mesenchymal cells (Osathanon et al. 2013). This quality of PDLSCs is associated with osteoblast differentiation and periodontal tissue remodeling (An et al. 2015; Hakki et al. 2014; Zhao and Liu 2014). Osteogenic differentiation of PDLSCs results from a complex molecular interplay involving the action of various transcription factors. In our study, we examined the effect of PERK overexpression and PERK knockout on osteogenic differentiation of PDLSCs. PERK is a novel cytokine for osteoblast differentiation and bone formation. PERK $^{-/-}$mice exhibited severe osteopenia involving decreases in the number of mature osteoblasts and bone thickness, and impaired osteoblast differentiation (Wei et al. 2008b). In addition, loss-of-function mutations of PERK in humans and mice cause several neonatal developmental defects, including diabetes, growth retardation, and multiple skeletal dysplasia (Biason-Lauber et al. 2002; Harding et al. 2012; Iyer et al. 2004; Lakshmanan et al. 2013). Similarly, ATF4deficient mice showed a remarkable reduction or delay in mineralization of bones (Yang et al. 2004). The phenotypes observed in bone tissues of $\mathrm{PERK}^{-1-}$ mice are very similar to those observed in bone tissues of ATF$4^{-/-}$mice. As is already known, ATF4 is a downstream target of PERK. The PERK-ATF4 signaling pathway is involved in osteoblastic differentiation of osteoblasts (Tanaka et al. 2014). In our study, the effect of the PERK-ATF4 pathway on osteogenic differentiation of hPDLSCs was explored. Our results indicated that this signaling pathway is involved in osteodifferentiation of hPDLSCs. The reasons for arriving at this conclusion are as follows: (1) The ATF4 mRNA level was reduced in PERK ${ }^{-/-}$hPDLSCs, and the expression of ATF4 was increased in Lenti-PERK hPDLSCs. (2) After osteogenic induction, the expression levels of osteogenesis-related genes were decreased in anti-PERK PDLSCs, while these levels were increased in PERK cells. (3) ALP activity and matrix mineralization were reduced in anti-PERK hPDLSCs. These findings support the suggestion that the PERK-eIF2 $\alpha$-ATF4 signaling pathway plays a role in osteodifferentiation of hPDLSCs in osteogenic cultures.

PERK is a type I transmembrane protein located in the ER, and PERK is a major transducer of the endoplasmic reticulum response (ERS) (Sood et al. 2000). Scheuner D found that PERK gene knockout cells were highly sensitive to ERS, while PERK knockout mice exhibited a series of serious defects, such as small size, bone dysplasia, and type
I diabetes (Back et al. 2009; Muaddi et al. 2010; Scheuner et al. 2006). When PERK is activated, it causes phosphorylation of eIF2 $\alpha$, which inhibits protein synthesis (Sood et al. 2000; Roobol et al. 2015). However, unlike most proteins, ATF4 escapes translational attenuation by eIF $2 \alpha$ phosphorylation because ATF4 has upstream open reading frames (ORFs) in its 5'-untranslated region. ATF4, one of the transcription factor members of the CREB family, is a major regulator of osteoblast differentiation and bone formation (Karpinski et al. 1992; Hamamura and Yokota 2007). In addition, ATF4 promotes osteogenic differentiation of PDLSCs by upregulating the expression of some osteogenic marker genes (Yang and Karsenty 2004). However, ATF4 and Runx2 synergistically regulate osteoblast differentiation and bone formation (Hagh et al. 2015; Nishimura 2011). Furthermore, some researchers found that upregulation of ATF4 may involve p-eIF2 $\alpha$ and PERK in the cardiovascular, kidney, and other tissues (Helenius 2001; Liu et al. 2015; Wang et al. 2014). Several studies have clearly indicated that ATF4 is an essential transcription factor for bone formation and terminal osteoblast differentiation. OCN and BSP are transcriptional targets of ATF4, which are osteoblast specific and markers for osteoblast differentiation (Ducy et al. 1996; Yeung et al. 2002). Studies have shown that the PERK-eIF2 $\alpha$-ATF4 signaling pathway is involved in osteoblastic differentiation of osteoblasts (Saito et al. 2011). Their findings indicated that the PERK-eIF2 $\alpha$-ATF4 signaling pathway plays a role in bone morphogenetic protein 2 (BMP2)-induced osteoblast differentiation and osteogenesis in culture (Saito et al. 2011). However, whether the PERKeIF $2 \alpha$-ATF4 signaling pathway is involved in osteogenic differentiation of PDLSCs is not known. In our previous study, we showed that PERK enhanced mechanical force-induced osteogenic differentiation in hPDLSCs (Yang et al. 2016). It is reasonable that PERK may function depending on the type of stem cells used and the physiological conditions applied. In this study, we sought to examine the mechanistic role of PERK in osteogenic differentiation of hPDLSCs. Our results indicated that the mRNA expression levels of ATF4 and other osteogenic genes were downregulated in anti-PERK hPDLSCs compared to untransfected cells, and there was a greater increase in PERK hPDLSCs compared to untransfected cells. These results indicated that osteoblast differentiation of hPDLSCs is enhanced by overexpression of PERK, since the expression of osteoblast-specific genes was strongly up-regulated. As is already known, hPDLSCs have been shown to express part of the phenotypic characteristics of osteoblasts in vitro (Gay et al. 2007; Kim et al. 2012). Our findings suggested that PERK may be a novel signaling pathway participating in osteoblast differentiation of hPDLSCs, which is important for bone remodeling.

Clinical observations have revealed that rapid and active alveolar bone remodeling followed by tooth movement does 
not occur unless normal healthy PDL surrounds the tooth root, and loss of the PDL subsequent to tooth extraction leads to resorption of the alveolar bone. Osteoclasts are the only cells that are responsible for bone re-absorption, while the formation and activity of osteoclasts are regulated by osteoblasts through expression of the osteoclast differentiation factor/RANKL. Thus, it is believed that differentiation of hPDLSCs to osteoblast-like cells plays an important role not only in bone formation but also in bone remodeling. Our study provided a novel insight that PERK is associated with differentiation of hPDLSCs. To develop more strategies, a detailed understanding of PERK targets and effects exerted by this signaling pathway on osteodifferentiation of hPDLSCs in vivo is necessary.

In summary, this study implied that PERK is essential in the process of differentiation of hPDLSCs into osteoblastlike cells. The PERK-eIF2 $\alpha$-ATF4 signaling pathway plays a role in osteodifferentiation of hPDLSCs in osteogenic cultures. This finding will contribute to a better understanding on the mechanism of periodontal remodeling via the PERK pathway.

Acknowledgements This study was supported by the National Natural Science Foundation of China (Grant Nos. 81800926 and 81771030).

\section{Compliance with ethical standards}

Conflict of interest The authors declare no conflict of interest.

Ethical approval hPDLSCs were obtained from premolar teeth extracted from young men (11-16 years old) for orthodontic reasons whose parents gave informed consent and were approved by the Ethics Committee of School and Hospital of Stomatology, Shandong University for the study.

Open Access This article is licensed under a Creative Commons Attribution 4.0 International License, which permits use, sharing, adaptation, distribution and reproduction in any medium or format, as long as you give appropriate credit to the original author(s) and the source, provide a link to the Creative Commons licence, and indicate if changes were made. The images or other third party material in this article are included in the article's Creative Commons licence, unless indicated otherwise in a credit line to the material. If material is not included in the article's Creative Commons licence and your intended use is not permitted by statutory regulation or exceeds the permitted use, you will need to obtain permission directly from the copyright holder. To view a copy of this licence, visit http://creativecommons.org/licenses/by/4.0/.

\section{References}

An S, Huang X, Gao Y, Ling J, Huang Y, Xiao Y (2015) FGF-2 induces the proliferation of human periodontal ligament cells and modulates their osteoblastic phenotype by affecting Runx 2 expression in the presence and absence of osteogenic inducers. Int $\mathrm{J}$ Mol Med 36:705-711
Back SH, Scheuner D, Han J, Song B, Ribick M, Wang J, Gildersleeve RD, Pennathur S, Kaufman RJ (2009) Translation attenuation through eIF2alpha phosphorylation prevents oxidative stress and maintains the differentiated state in beta cells. Cell Metab 10:13-26

Biason-Lauber A, Lang-Muritano M, Vaccaro T, Schoenle EJ (2002) Loss of kinase activity in a patient with Wolcott-Rallison syndrome caused by a novel mutation in the EIF2AK3 gene. Diabetes 51:2301-2305

Ducy P, Geoffroy V, Karsenty G (1996) Study of osteoblast-specific expression of one mouse osteocalcin gene: characterization of the factor binding to OSE2. Connect Tissue Res 35:7-14

Gay IC, Chen S, MacDougall M (2007) Isolation and characterization of multipotent human periodontal ligament stem cells. Orthod Craniofac Res 10:149-160

Hagh MF, Noruzinia M, Mortazavi Y, Soleimani M, Kaviani S, Abroun S, Dehghani Fard A, Mahmoodinia M (2015) Different methylation patterns of RUNX2, OSX, DLX5 and BSP in osteoblastic differentiation of mesenchymal stem cells. Cell J 17:71-82

Hai T, Hartman MG (2001) The molecular biology and nomenclature of the activating transcription factor/cAMP responsive element binding family of transcription factors: activating transcription factor proteins and homeostasis. Gene 273:1-11

Hakki SS, Bozkurt B, Hakki EE, Kayis SA, Turac G, Yilmaz I, Karaoz E (2014) Bone morphogenetic protein-2, -6, and -7 differently regulate osteogenic differentiation of human periodontal ligament stem cells. J Biomed Mater Res B 102:119-130

Hamamura K, Yokota H (2007) Stress to endoplasmic reticulum of mouse osteoblasts induces apoptosis and transcriptional activation for bone remodeling. FEBS Lett 581:1769-1774

Harding HP, Zhang Y, Bertolotti A, Zeng H, Ron D (2000) Perk is essential for translational regulation and cell survival during the unfolded protein response. Mol Cell 5:897-904

Harding HP, Zyryanova AF, Ron D (2012) Uncoupling proteostasis and development in vitro with a small molecule inhibitor of the pancreatic endoplasmic reticulum kinase, PERK. J Biol Chem 287:44338-44344

Helenius A (2001) Quality control in the secretory assembly line. Philos Trans R Soc Lond B 356:147-150

Huang G, Yao J, Zeng W, Mizuno Y, Kamm KE, Stull JT, Harding HP, Ron D, Muallem S (2006) ER stress disrupts $\mathrm{Ca}^{2+}$-signaling complexes and $\mathrm{Ca}^{2+}$ regulation in secretory and muscle cells from PERK-knockout mice. J Cell Sci 119:153-161

Iyer S, Korada M, Rainbow L, Kirk J, Brown RM, Shaw N, Barrett TG (2004) Wolcott-Rallison syndrome: a clinical and genetic study of three children, novel mutation in EIF2AK3 and a review of the literature. Acta Paediatr 93:1195-1201

Karpinski BA, Morle GD, Huggenvik J, Uhler MD, Leiden JM (1992) Molecular cloning of human CREB-2: an ATF/CREB transcription factor that can negatively regulate transcription from the cAMP response element. Proc Natl Acad Sci USA 89:4820-4824

Kim SS, Kwon DW, Im I, Kim YD, Hwang DS, Holliday LS, Donatelli RE, Son WS, Jun ES (2012) Differentiation and characteristics of undifferentiated mesenchymal stem cells originating from adult premolar periodontal ligaments. Korean J Orthod 42:307-317

Komori T (2006) Regulation of osteoblast differentiation by transcription factors. J Cell Biochem 99:1233-1239

Lakshmanan AP, Harima M, Suzuki K, Soetikno V, Nagata M, Nakamura T, Takahashi T, Sone H, Kawachi H, Watanabe K (2013) The hyperglycemia stimulated myocardial endoplasmic reticulum (ER) stress contributes to diabetic cardiomyopathy in the transgenic non-obese type 2 diabetic rats: a differential role of unfolded protein response (UPR) signaling proteins. Int J Biochem Cell Biol 45:438-447 
Liu Z, Lv Y, Zhao N, Guan G, Wang J (2015) Protein kinase R-like ER kinase and its role in endoplasmic reticulum stress-decided cell fate. Cell Death Dis 6:e1822

Muaddi H, Majumder M, Peidis P, Papadakis AI, Holcik M, Scheuner D, Kaufman RJ, Hatzoglou M, Koromilas AE (2010) Phosphorylation of eIF2 $\alpha$ at serine 51 is an important determinant of cell survival and adaptation to glucose deficiency. Mol Biol Cell 21:3220-3231

Nagatomo K, Komaki M, Sekiya I, Sakaguchi Y, Noguchi K, Oda S, Muneta T, Ishikawa I (2006) Stem cell properties of human periodontal ligament cells. J Periodontal Res 41:303-310

Nishimura R (2011) Bone and calcium update; bone research update. Regulatory mechanisms in osteoblast differentiation. Clin Calcium 21:103-112

Osathanon T, Ritprajak P, Nowwarote N, Manokawinchoke J, Giachelli C, Pavasant P (2013) Surface-bound orientated Jagged-1 enhances osteogenic differentiation of human periodontal ligament-derivedmesenchymal stem cells. J Biomed Mater Res A 101:358-367

Roobol A, Roobol J, Bastide A, Knight JR, Willis AE, Smales CM (2015) p58IPK is an inhibitor of the eIF2 $\alpha$ kinase GCN2 and its localization and expression underpin protein synthesis and ER processing capacity. Biochem J 465:213-225

Saito A, Ochiai K, Kondo S, Tsumagari K, Murakami T, Cavener DR, Imaizumi K (2011) Endoplasmic Reticulum Stress response mediated by the PERK-eIF2 $\alpha$-ATF4 pathway is involved in osteoblast differentiation induced by BMP2. J Biol Chem 286:4809-4818

Scheuner D, Patel R, Wang F, Lee K, Kumar K, Wu J, Nilsson A, Karin M, Kaufman RJ (2006) Double-stranded RNA-dependent protein kinase phosphorylation of the alpha-subunit of eukaryotic translation initiation factor 2 mediates apoptosis. J Biol Chem 281:21458-21468

Seo BM, Miura M, Gronthos S, Bartold PM, Batouli S, Brahim J, Young M, Robey PG, Wang CY, Shi S (2004) Investigation of multipotent postnatal stem cells from human periodontal ligament. Lancet 364:149-155

Silvério KG, Rodrigues TL, Coletta RD, Benevides L, Da Silva JS, Casati MZ, Sallum EA, Nociti FH Jr (2010) Mesenchymal stem cell properties of periodontal ligament cells from deciduous and permanent teeth. J Periodontol 81:1207-1215

Sood R, Porter AC, Ma K, Quilliam LA, Wek RC (2000) Pancreatic eukaryotic initiation factor-2alpha kinase (PEK) homologues in humans, Drosophila melanogaster and Caenorhabditis elegans that mediate translational control in response to endoplasmic reticulum stress. Biochem J 346:281-293

Tanaka K, Kaji H, Yamaguchi T, Kanazawa I, Canaff L, Hendy GN, Sugimoto T (2014) Involvement of the osteoinductive factors,
Tmem119 and BMP-2, and the ER stressresponse PERK-eIF2 $\alpha$ ATF4 pathway in the commitment of myoblastic into osteoblastic cells. Calcif Tissue Int 94:454-464

Wang H, Xu X, Fassett J, Kwak D, Liu X, Hu X, Falls TJ, Bell JC, Li H, Bitterman P et al (2014) Double-stranded RNA-dependent protein kinase deficiency protects the heart from systolic overloadinduced congestive heart failure. Circulation 129:1397-1406

Wei F, Wang C, Zhou G, Liu D, Zhang X, Zhao Y, Zhang Y, Yang Q (2008a) The effect of centrifugal force on the mRNA and protein levels of ATF4 in cultured human periodontal ligament fibroblasts. Arch Oral Biol 53:35-43

Wei J, Sheng X, Feng D, McGrath B, Cavener DR (2008b) PERK is essential for neonatal skeletal development to regulate osteoblast proliferation and differentiation. J Cell Physiol 217:693-707

Xiao G, Jiang D, Ge C, Zhao Z, Lai Y, Boules H, Phimphilai M, Yang X, Karsenty G, Franceschi RT (2005) Cooperative interactions between activating transcription factor 4 and Runx $2 / \mathrm{Cbfa} 1$ stimulate osteoblast-specific osteocalcin gene expression. J Biol Chem 280:30689-30696

Yang X, Karsenty G (2004) ATF4, the osteoblast accumulation of which is determined post-translationally, can induce osteoblastspecific gene expression in non-osteoblastic cells. J Biol Chem 279:47109-47114

Yang X, Matsuda K, Bialek P, Jacquot S, Masuoka HC, Schinke T, Li L, Brancorsini S, Sassone-Corsi P, Townes TM et al (2004) ATF4 is a substrate of RSK2 and an essential regulator of osteoblast biology; implication for Coffin-Lowry Syndrome. Cell 117:387-398

Yang SY, Wei FL, Hu LH, Wang CL (2016) PERK-eIF2 $\alpha$-ATF4 pathway mediated by endoplasmic reticulum stress response is involved in osteodifferentiation of human periodontal ligament cells under cyclic mechanical force. Cell Signal 28:880-886

Yeung F, Law WK, Yeh CH, Westendorf JJ, Zhang Y, Wang R, Kao C, Chung LW (2002) Regulation of human osteocalcin promoter in hormone-independent human prostate cancer cells. J Biol Chem 277:2468-7640

Zhao BJ, Liu YH (2014) Simvastatin induces the osteogenic differentiation of human periodontal ligament stem cells. Fundam Clin Pharmacol 28:583-592

Publisher's Note Springer Nature remains neutral with regard to jurisdictional claims in published maps and institutional affiliations. 